\title{
The Dangers of Dilution When Measuring Water Based Pharmaceutical Suspensions and How Nanoflex Overcomes these Obstacles
}

\section{David Pugh*}

Date of Receipt- 28/06/2017
Date of Revision- 09/07/2017

Date of Acceptance- 17/07/2017

\section{Address for}

Correspondence

Microtrac $\mathrm{GmbH}$, Campus

Fichtenhain 42, 47807 Krefeld, Germany

E-mail: david.pugh@microtrac.

com

\begin{abstract}
Dynamic Light Scattering (DLS) has been an important tool for determining particle size distributions in fine particulate material suspensions, micro emulsions and Nano-scale matter in general for 50 years. Many Nano scale materials are measured in non-aqueous media and as long as we know the viscosities in these media at 2 different temperatures, then the analysis are relatively simple. However most pharmaceutical suspensions are water based and the key danger is not in the sampling but the chemistry.
\end{abstract}

When measuring the size of particles, a study of their zeta potential in advance is extremely important as a high zeta potential is indicative of a stable product whilst a low zeta potential is indicative of an unstable product likely to agglomerate. When two particles have a high zeta potential they repel each other like 2 negative or positive magnets so they are not attracted to each other thus leading to a stable solution. When a sample is diluted dramatic changes to its zeta potential can occur especially when the dilution causes the zeta potential to approach the Iso Electric Point (IEP-0 $\mathrm{m}$ Volts). There are a number of effects which may cause the zeta potential to decrease towards 0 $\mathrm{mV}$ after dilution; we will cover $\mathrm{pH}$, salt concentration and polyelectrolyte or surfactant concentration. There are 2 approaches to take when encountering this problem, The first approach requires full knowledge of the suspensions $\mathrm{pH}$, salt concentration and volume \% concentration of the surfactant such that care in dilution may be effected (Prevention). The second involves technological advances (Prevention and Cure) which are unique and employs a method which in most pharmaceutical applications renders the need to dilute redundant. By measuring the suspension at full concentration, all potential chemistry problems are negated.

Keywords: Scattering, Particle, Suspension, Dilution, Concentration, Pharmaceutical, Potential.

\section{INTRODUCTION}

Brownian motion is the principle behind dynamic light scattering and is very simple in its concept. In the case of a water based suspension, the water molecules (size 0.3 $\mathrm{nm})$ collide with particles causing the particle to move with a certain velocity. The larger the particle the slower the velocity due to its greater mass and in fact when the particle is so large, that its velocity due to sedimentation is greater than the collision velocity due to Brownian motion determines the upper size limit to which DLS can measure particles. The lower end of measurement is determined by the size of the molecules colliding with the particles ${ }^{1,2}$. As the size of a water molecule is roughly $0.3 \mathrm{~nm}$, it is obvious that the lower limit of detection is above this size. A depiction of the particles random movement is shown in Figure 1.

Historically two modes of optical measurement by DLS have commonly been used, self-beating (Homodyne) detection (1960s) and controlled reference or (Heterodyne) detection (1980s) ${ }^{3}$. Homodyne detection (PCS) involves monitoring the light scattering signals (photons) and 
analyzing by an autocorrelation function, whereas the Heterodyne method directly measures the frequency shift power spectrum (FPS) (Figure 2).

The optical arrangement in PCS systems have used Helium Neon lasers, correlators and Non Negative Least Squares (NNLS) software fitting routines to determine a result. The scattering capture angles were initially set at 90 degrees and later at lower and higher angles and the path length is $10 \mathrm{~mm}$ typically. The optical arrangement in Heterodyne FPS is entirely only measuring the backscattered light (180 degrees). The solid state laser diode is in intimate contact with the particles and measures the back scattering from particles up to 300 microns $(0.3 \mathrm{~mm})$ into the media and is directly collected by a detector a detector. As the path length is 30 times (10 mm compared to 0.3 $\mathrm{mm}$ ) less on the Heterodyne FPS (Figure 3) method compared to the PCS method, we can measure much higher concentrations which allows us to measure fully concentrated product in the processes without any need to dilute and the problems that can ensue from dilution ${ }^{1}$. Because no dilution is required FPS paves the way to thus measure lab samples by placing the probe directly into the sample container. In addition, it can be integrated into fully automated at-line systems such as Chemspeed, which measures sample parameters at once without supervision. Online systems involve taking a bypass sample from a reactor vessel with probe inserted in the bypass, but ultimately the best way forward is to measure the suspension in-line directly in the reactor. As we are measuring the velocity of the particles to determine size, it is necessary that we only measure the Brownian motion and not any created motion from stirring or natural flow. For this reason, we have created a sheath to cover the probe. The end of the sheath will of course have liquid suspension moving around it, but a certain distance up the sheath there will be a point where there is no movement apart from that of Brownian motion. This is where the tip of the probe will be and this will allow us to measure undiluted suspensions in real time without dilution. The $180^{\circ}$ DLS backscattering (Figure 4) is especially suitable for direct in-line use in reactors, because the handling of the measurement probe head with a diameter of 8 $\mathrm{mm}$ is just as easy as a $\mathrm{pH}$ head.

\section{METHODS}

What about the chemistry of dilution, why is it so potentially problematic? The answer lies purely in the instabilities created within the suspension when it is diluted and its zeta potential changes.

The most important parameter to affect the stability of a suspension is the $\mathrm{pH}$. In Figure 5 we can see dramatic effect on the zeta potential when performing a $\mathrm{pH}$ titration.

The product is stable in low $\mathrm{pH}$ acidic dilutions, but as the $\mathrm{pH}$ increases the product reaches the isoelectric point and becomes very unstable and agglomerates, as is shown in Figure 6 where the size of the suspension increases from $150 \mathrm{nM}$ to greater than $1000 \mathrm{nM}$. It is thus very important to know the original $\mathrm{pH}$ of your suspension and dilute accordingly with a solution of that $\mathrm{pH}$.

The second most important parameter to monitor and control is that of surfactant concentration. Yet again if any dilution is to take place the surfactant concentration in the dilution must remain the same. Surfactant can provide stability in a suspension and any dilution of the surfactant in the suspension can reduce the zeta potential and cause the particles to agglomerate.

The third parameter which must be considered is that of conductivity or salt concentration. In this case an addition of salt causes the zeta potential to plummet, so yet again, upon dilution, the suspension's original salt concentration or conductivity must be maintained. This added difficulty is demonstrated in Figure 7.

\section{RESULTS}

The problems of not caring about the chemistry of dilution are shown in the comparison result (Figure 8) of a material which when measured undiluted revealed a bimodal distribution with a shoulder at the fine end of the range. When diluted 50/50, a large quantity of fines agglomerated to provide a monomodal distribution which would be misleading to most researchers.

In the following examples (Figure 9) we can see that the Nanoflex FPS technology which is not constrained by software models or predetermined by the user to expect a certain type of curve, yields accurate results unobtainable by standard PCS methods which model a seemingly perfect bell curve ${ }^{2}$.

In Figure 10, 2 populations are missed due to curve fitting routines which FPS does not need or use. We reveal a population from 15 to $100 \mathrm{~nm}$ which is completely missed by the PCS result on the right. In fact the post 1 micron population is also missed by the PCS analysis.

In the final example in Figure 11, the material has gone through a 0.2 micron filter which is demonstrated by the maximum size of 0.2 micron in the FPS Nanoflex result on the left but not the PCS result on the right. The lower limit of $20 \mathrm{~nm}$ is accurately determined by both the FPS and PCS systems but the PCS result on 
the right shows a seemingly perfect bell curve with a peak at $80 \mathrm{~nm}$ whereas the reality is depicted by the Nanoflex FPS result on the left where the true peak at $35 \mathrm{~nm}$ not $80 \mathrm{~nm}$ is located.

\section{DISCUSSION}

So we have a technology which can measure suspensions without dilution, which is an obvious advantage to researchers, QC and QA managers who have not known of the pitfalls of dilution before. Frankly do they have the time to correct the situation by performing all the titration tests I have suggested in order to fully understand and dilute the suspension with a diluent that is as close as possible to the original formulation? With the Nanoflex-No dilution system, they can measure the suspension as it is without creating a new agglomerated suspension which bears no reality to the original size.

More importantly, what does a no dilution system provide you not in the future but now?

Well first of all it is now possible to measure your suspensions on-line with or without dilution as is shown in (Figure 12) ${ }^{3}$.

In addition we can take it further and measure directly in-line, in a reactor or indeed any kind of vessel where you want to measure accurately undiluted and in real time as is shown in the formulation vessels shown in the pictures 8-10 below and the (Figure 13) where a material was decreased in size and monitored for 120 minutes until the correct size was attained. We now have eyes in the process for nano-materials.

\section{CONCLUSION}

The knowledge to effect good measurements on nanomaterials has been around for a long time but in many particle size measurements the thought that is needed when diluting suspensions has not been used and is often ignored as a minor inconvenience. The reality is that even a change of water supply has the potential to change the result. Microtrac by introducing the Nanoflex technology has enabled users to measure their samples undiluted and hence attain accurate results. Some materials size changes when there is a change of temperature or pressure, so the ability to measure either on-line in a bypass or in situ in the process vessel with no dilution is truly a "Holy Grail” for Research Scientists.

\section{REFERENCES}

1. Freud PJ. Nanoparticle sizing: Dynamic light scattering. Microtrac Application Notes. 2007;1-7.

2. Freeland B, McCann R, Bagga1 $\mathrm{K}$, et al. Nanoparticle fabrication via pulsed laser ablation in liquid: A step towards production scale-up.
2014.

3. Benen TD, Trainer M, Paul J. Freud nanoparticle sizing 2.0: Dynamic light scattering in the frequency spectrum mode. 2014. 

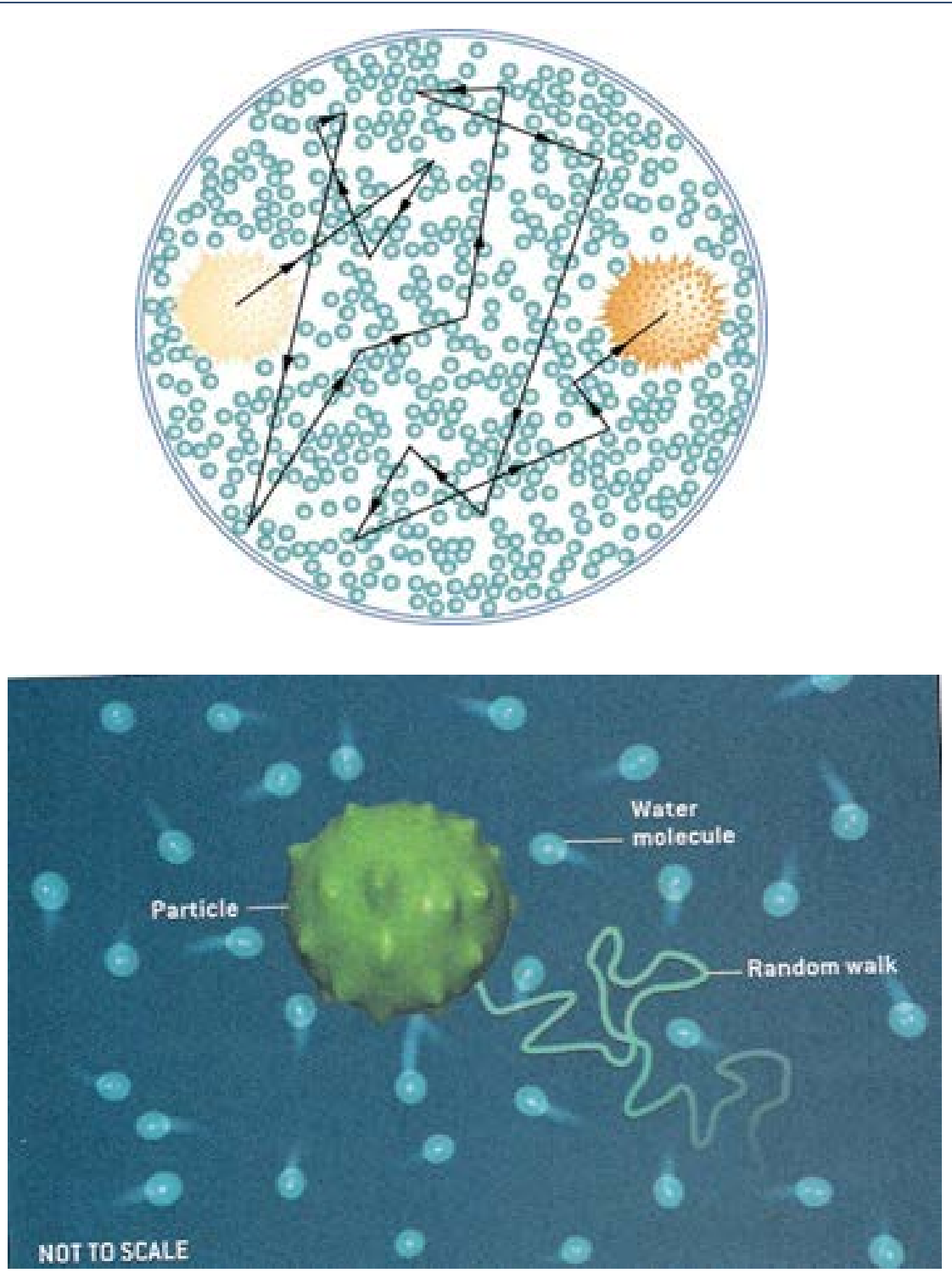

Figure 1. Depiction of the particles random movement

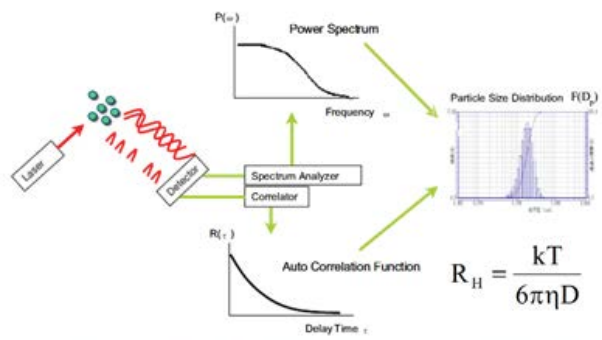

Homodyne method Heterodyne method (Nanotrac)

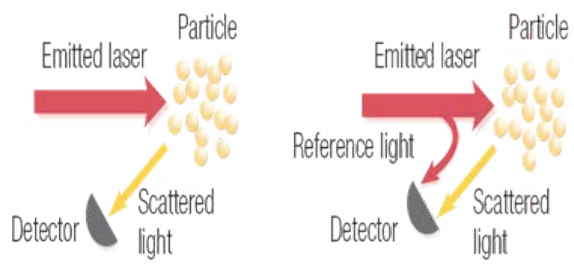

Figure 2. Depiction of the self-beating (homodyne) detection and controlled reference (heterodyne) detection 


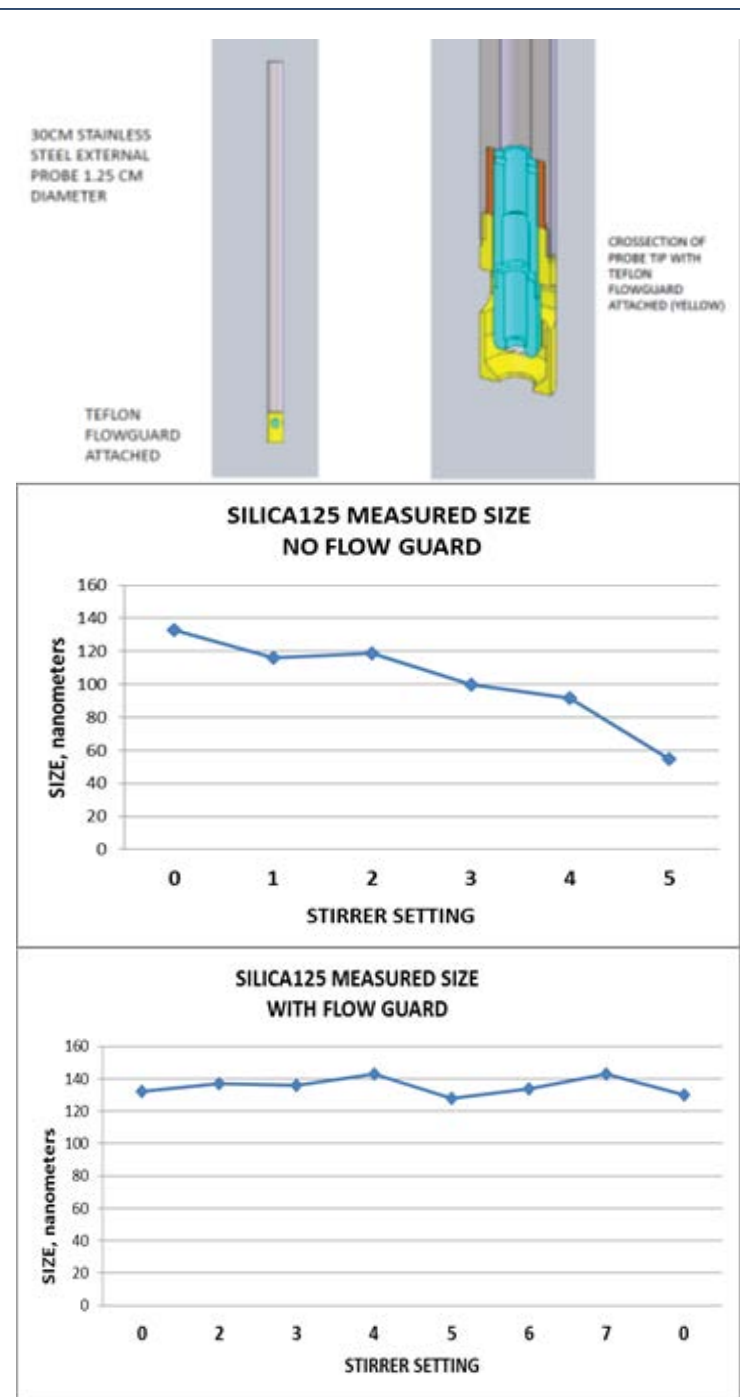

Figure 3. Heterodyne FPS method compared to the PCS method

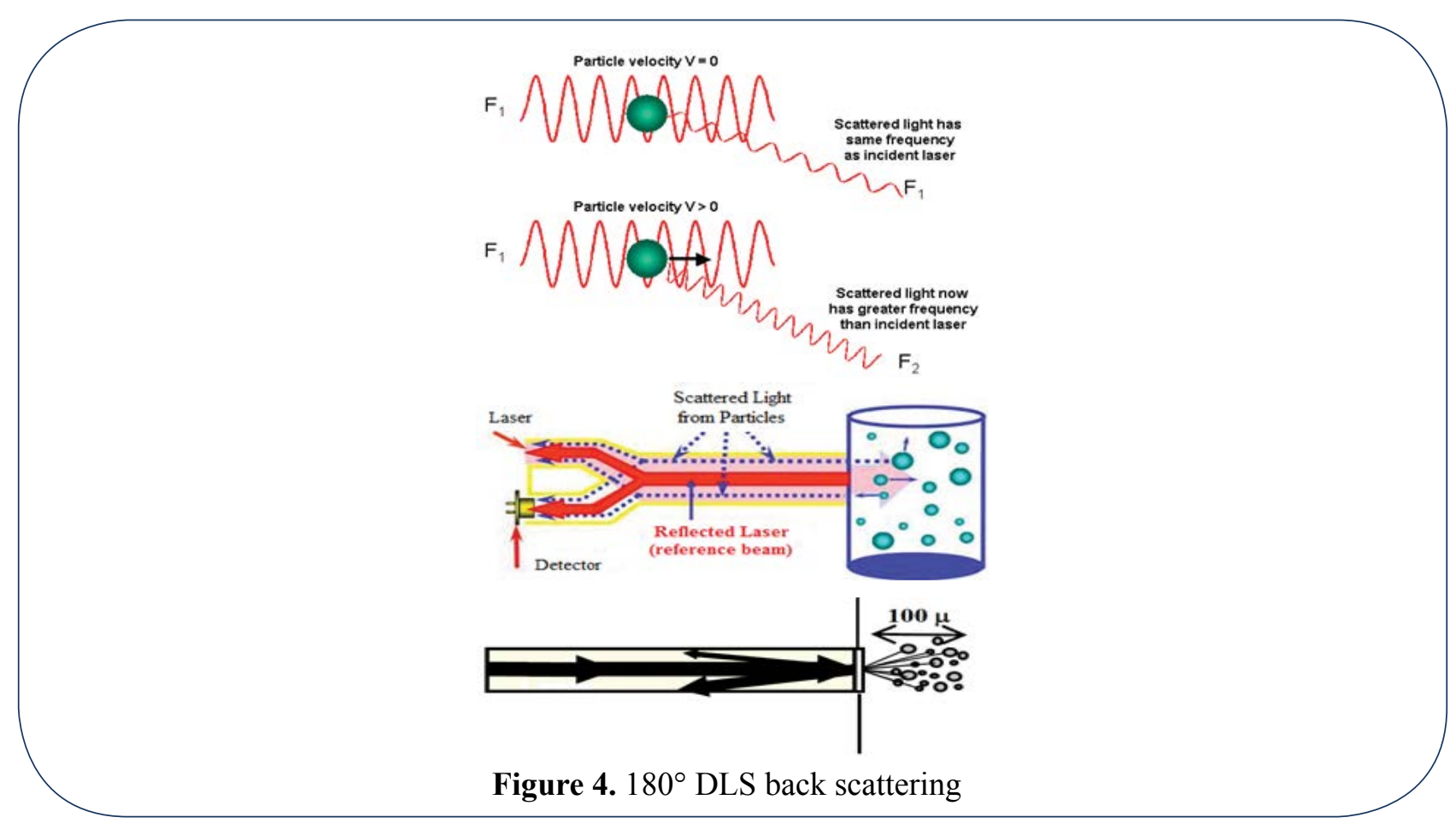



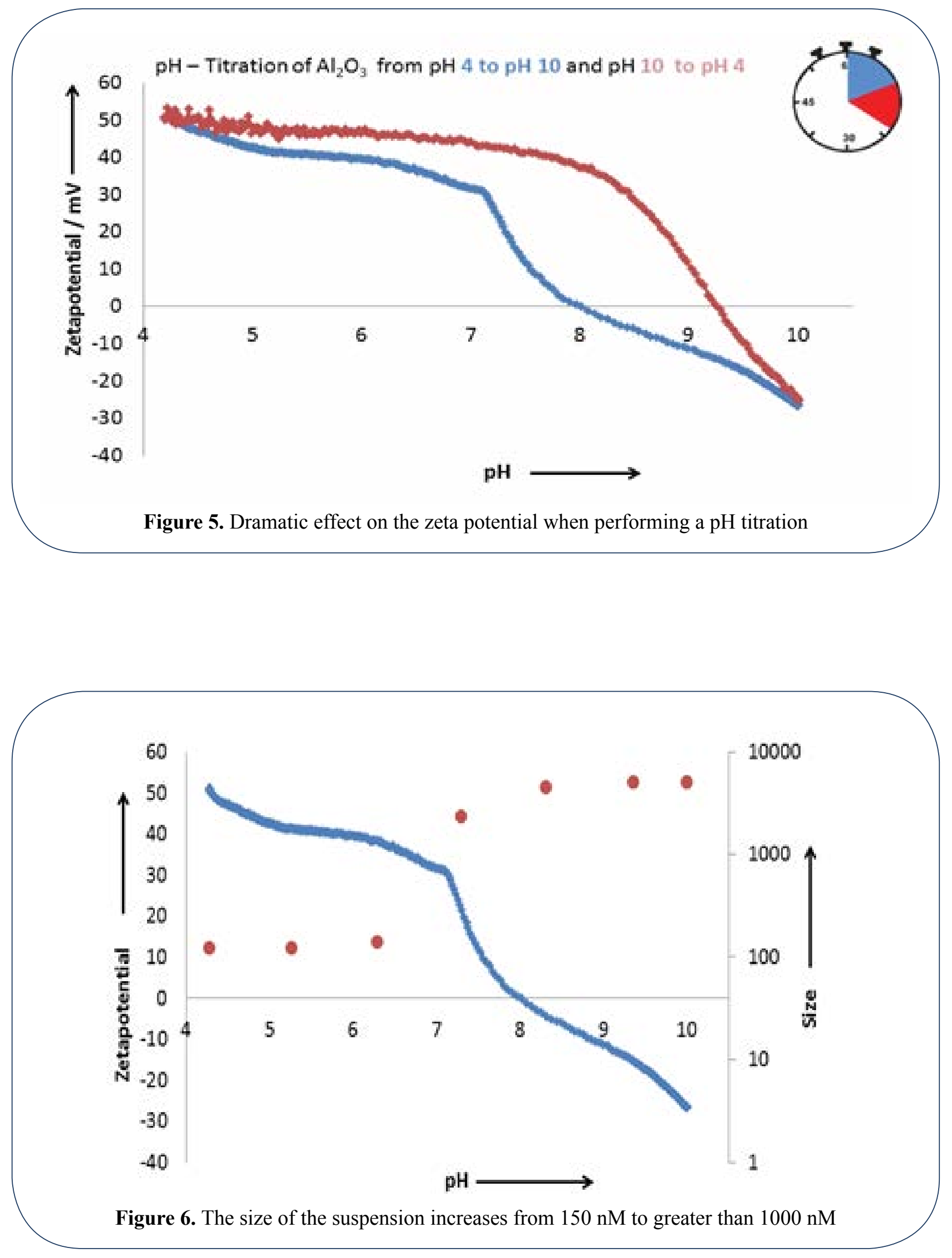


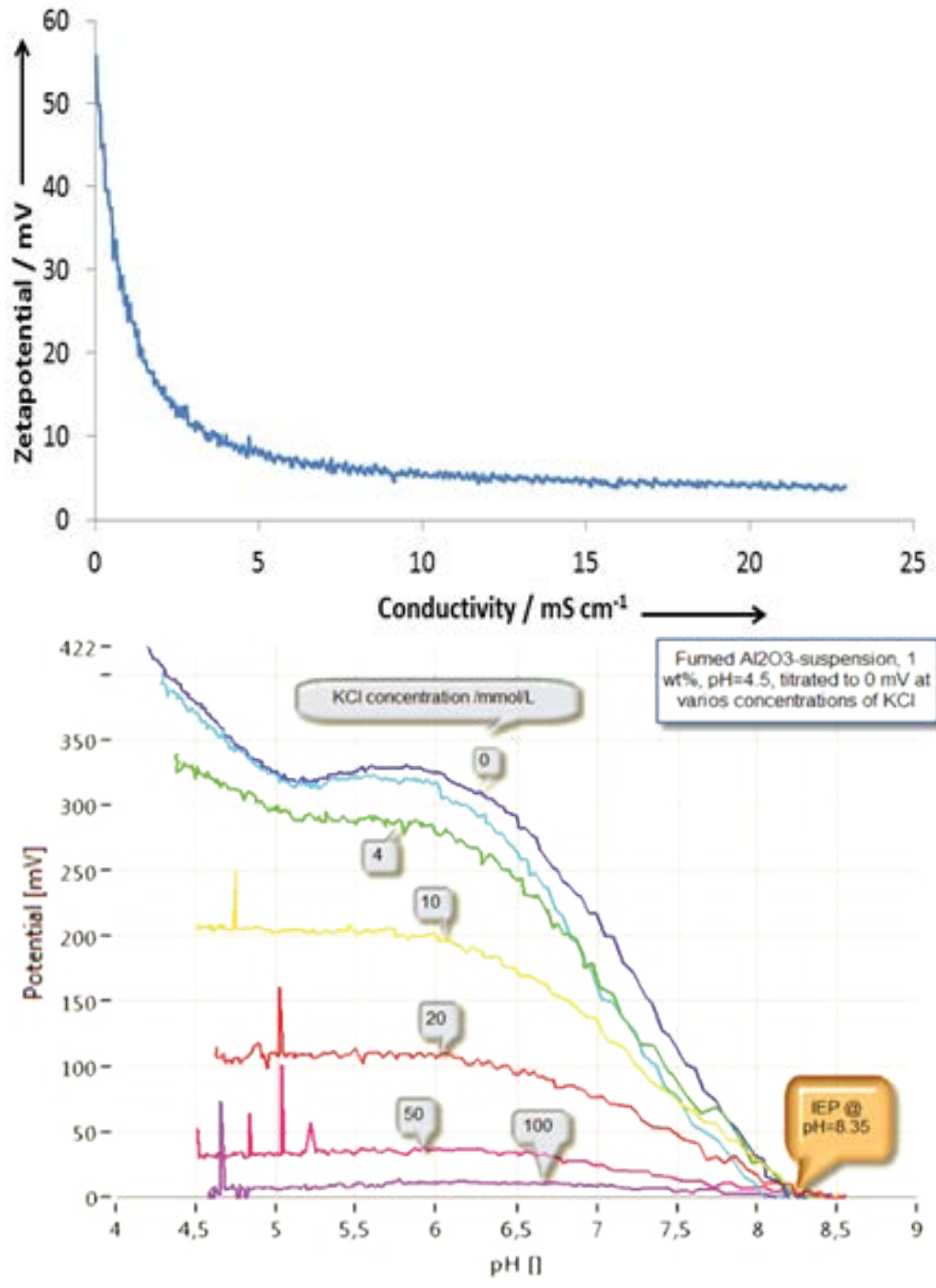

Figure 7. Zeta potential drops at high speed upon dilution

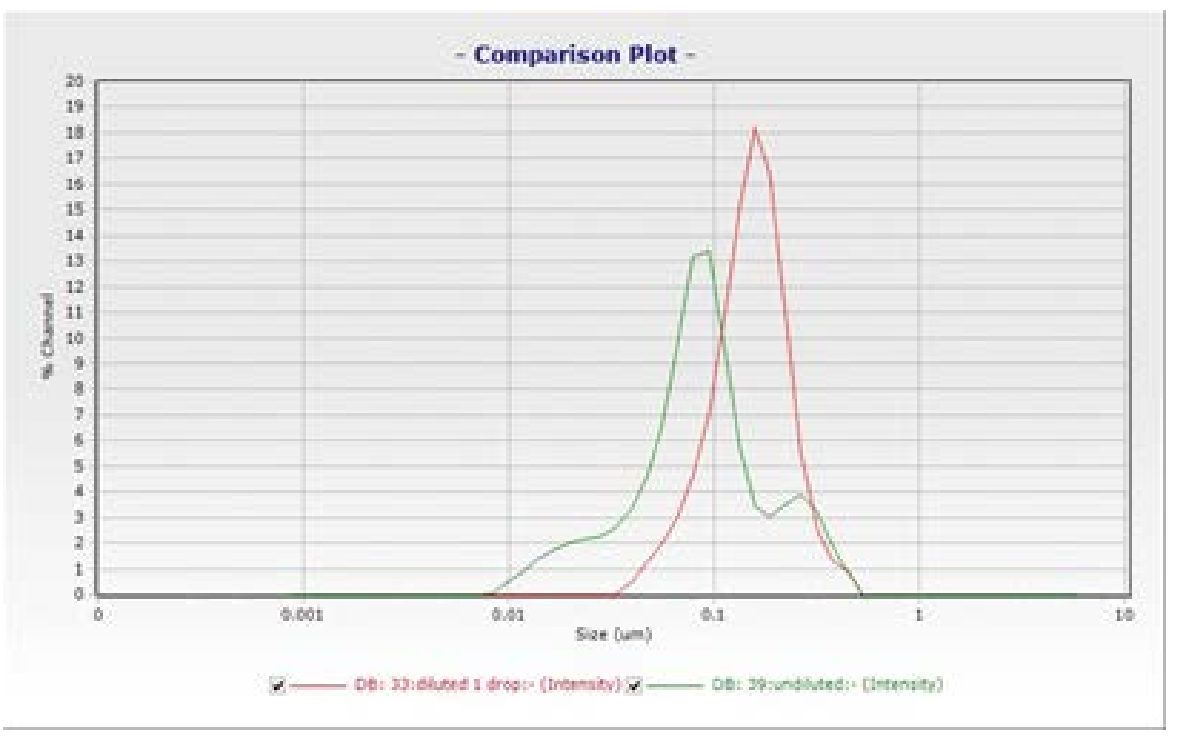

Figure 8. Comparison result of a material which when measured undiluted 

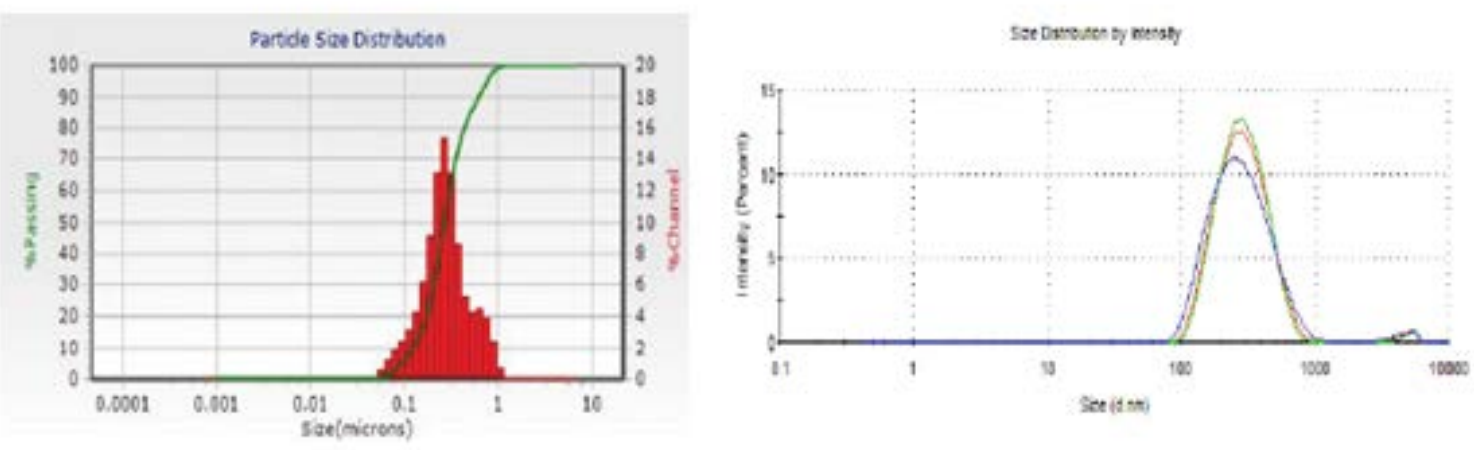

Figure 9. Results obtained by Nanoflex FPS technology on the left and PCS on the right
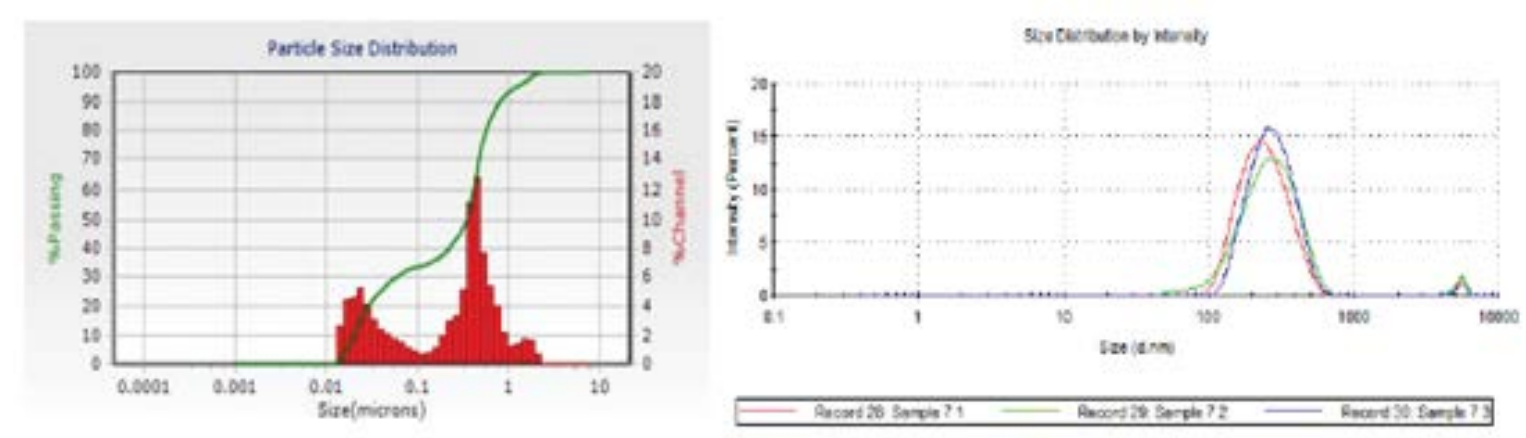

Figure 10. A population from 15 to $100 \mathrm{~nm}$ which is completely missed by the PCS result on the right
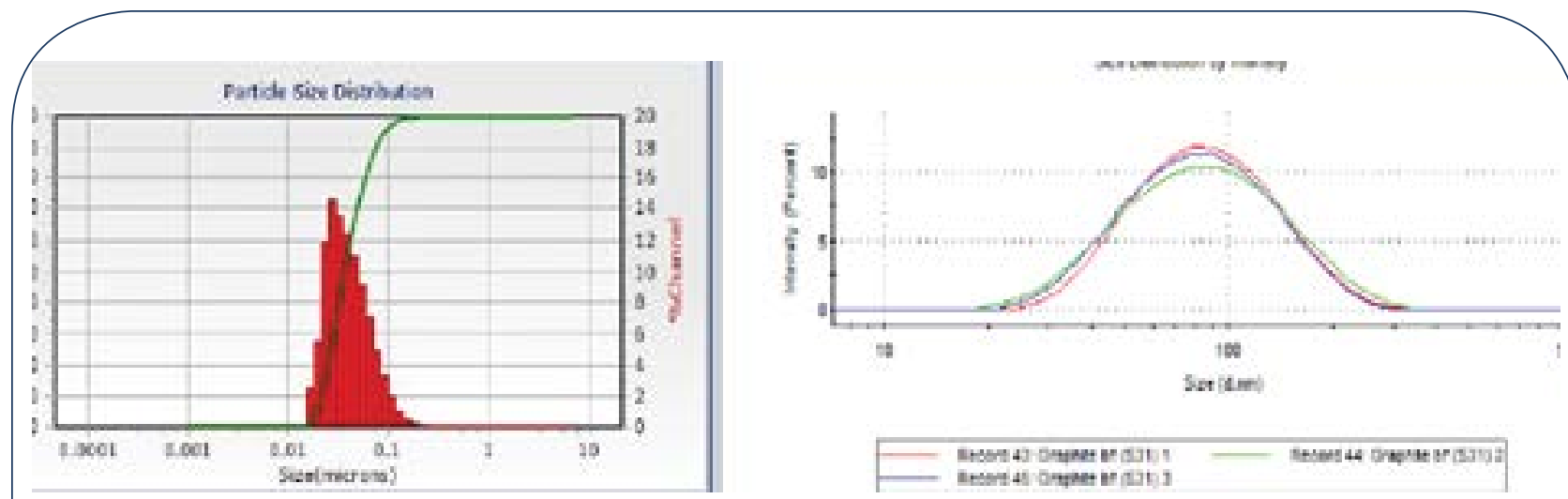

Figure 11. The material has gone through a 0.2 micron filter which is demonstrated by the maximum size of 0.2 micron in the FPS nanoflex result on the left but not the PCS result on the right 

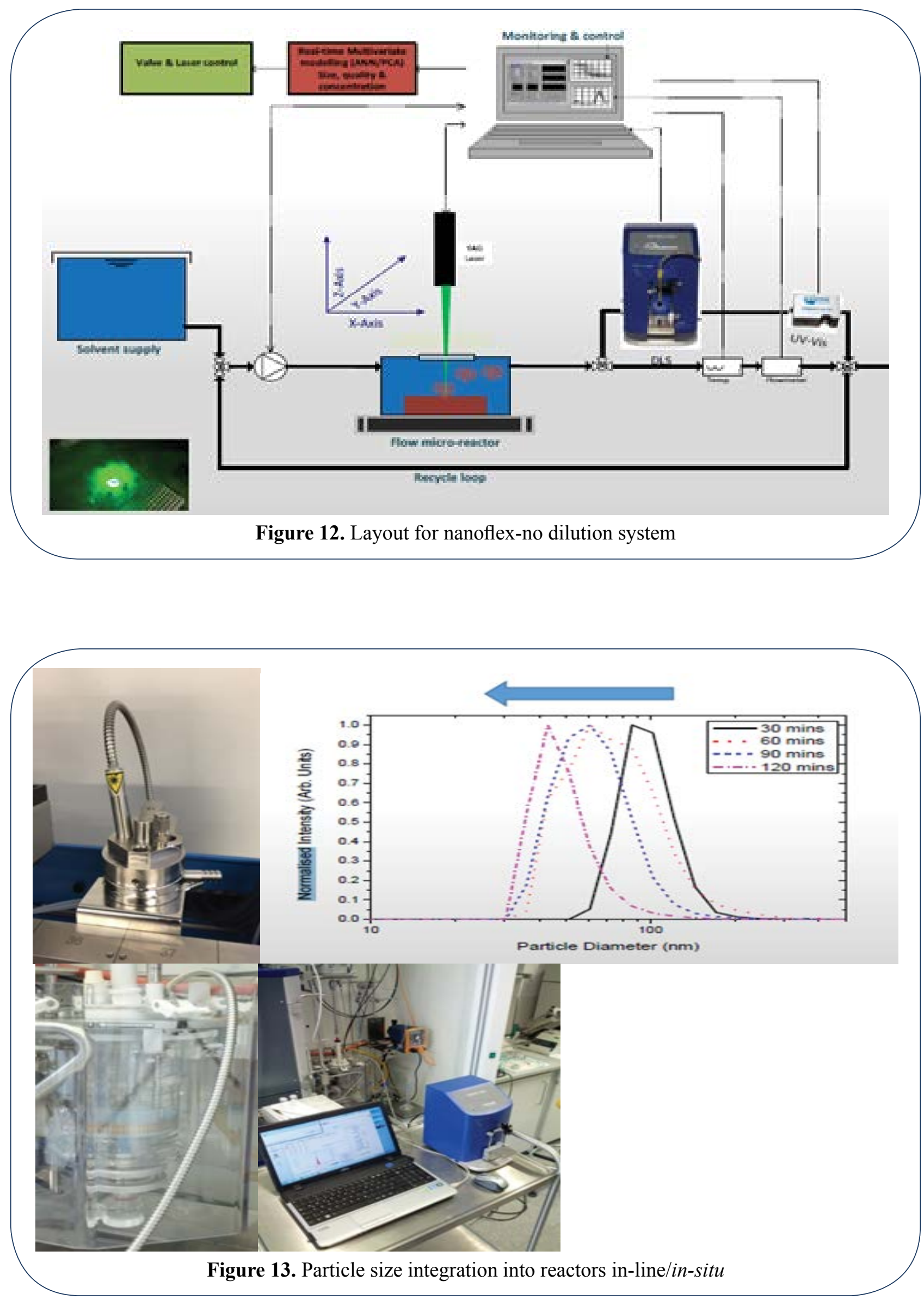